\title{
IMMANANTS, SCHUR FUNCTIONS, AND THE MACMAHON MASTER THEOREM
}

\author{
I. P. GOULDEN AND D. M. JACKSON
}

(Communicated by Jeffry N. Kahn)

\begin{abstract}
The relationship between the immanant and the Schur symmetric function is examined. Two expressions for the immanant are given in terms of the determinant. Generalisations include Foata and Zeilberger's $\beta$-extension of the MacMahon Master theorem. The relationships to some little known results of Littlewood and to idempotents constructed by Young are given.
\end{abstract}

\section{IISTRODUCTION}

For the symmetric group $\mathfrak{S}_{n}$ on $n$ symbols, let $\chi^{\lambda}(\sigma)$ denote the value, at $\sigma$, of the character $\chi^{\lambda}$ of the irreducible representation associated with the conjugacy class indexed by the partition $\lambda$ of $n$. The $\lambda$ th immanant of the $n \times n$ matrix $\mathbf{A}$, with $(i, j)$-element $a_{i, j}$, is defined by

$$
\operatorname{Imm}_{\lambda} \mathbf{A}=\sum_{\sigma \in \mathfrak{S}_{n}} \chi^{\lambda}(\sigma) \prod_{i=1}^{n} a_{i, \sigma(i)} .
$$

For the purposes of this paper, $a_{1,1}, a_{1,2}, \ldots, a_{n, n}$ are commutative indeterminates. $\operatorname{Imm}_{\left[1^{n}\right]} \mathbf{A}=\operatorname{det} \mathbf{A}$ and $\operatorname{Imm}_{[n]} \mathbf{A}=$ per $\mathbf{A}$, so $\operatorname{Imm}_{\lambda} \mathbf{A}$ is a multilinear function that interpolates between the determinant and the permanent. Some of the combinatorial properties of immanants were considered in [3].

The purpose of this paper is to examine the relationship between the Schur function and the immanant. Section 1 gives the necessary background on the ring of symmetric functions. In $\S 2$, we give a result expressing the immanant of A as the coefficient of $z_{1} \cdots z_{n}$ in the determinant of a designated matrix. It is shown that this gives, in $\S 3$, a generalisation of the MacMahon Master theorem [9]. We also show that the coefficient of $z_{1}^{k_{1}} \cdots z_{n}^{k_{n}}$ in the same determinant is expressible as the immanant of a matrix readily constructible from $\mathbf{A}$. A further extension, in which cycles are marked, yields a generalisation of Foata and Zeilberger's [1] $\beta$-extension of the MacMahon Master theorem. The connexion of these results with those of Littlewood [6,7] is given in $\S 4$ and with the Young idempotents is given in $\S 5$.

Received by the editors September 27, 1990 and, in revised form, December 20, 1990.

1980 Mathematics Subject Classification (1985 Revision). Primary 15A15; Secondary 05A15.

This work was supported by the Natural Sciences and Engineering Research Council of Canada grants A-8235 and A-8907. 
The reader is referred to [8] for further details on symmetric functions and to [2] for further information about Young's idempotents.

The following notation is needed. If $\lambda$ is a partition of $n$, then we write $\lambda \vdash n$. The number of parts of $\lambda$ is denoted by $l(\lambda)$. We also write $\lambda=$ $\left[1^{i_{1}} 2^{i_{2}} \ldots n^{i_{n}}\right]$, where $\lambda$ has $i_{j}$ parts equal to $j$, for $j=1, \ldots, n$. The conjugate of the partition $\lambda=\left(\lambda_{1}, \lambda_{2}, \ldots\right)$ is denoted by $\tilde{\lambda}$. The cycle-type of $\sigma \in \mathfrak{S}_{n}$ is $\tau(\sigma)=\left(i_{1}, i_{2}, \ldots\right)$, where $\sigma$ has $i_{j}$ cycles of length $j$. Since $\chi^{\lambda}$ is a class function, $\chi_{\mu}^{\lambda}$ denotes $\chi^{\lambda}(\sigma)$ where $\tau(\sigma)=\mu$. Let $\delta_{n}=(n-1, n-2, \ldots, 1,0)$.

Let $\mathrm{R}$ be a commutative ring, and let $\mathbf{z}=\left(z_{1}, \ldots, z_{n}\right), \mathbf{1}_{n}=(1, \ldots, 1), \mathbf{0}_{n}$ $=(0, \ldots, 0)$, with $n$ components. Also let $\mathbf{k}=\left(k_{1}, \ldots, k_{n}\right)$, a vector of nonnegative integers. When no confusion arises, $\mathbf{1}_{n}$ and $\mathbf{0}_{n}$ are replaced by $\mathbf{1}$ and $\mathbf{0}$. Then $\mathbf{z}^{\mathbf{k}}$ denotes $z_{1}^{k_{1}} \cdots z_{n}^{k_{n}}$ and $\left[\mathbf{z}^{\mathbf{k}}\right] f$ denotes the coefficient of $\mathbf{z}^{\mathbf{k}}$ in $f \in \mathrm{R}[[\mathbf{z}]]$. Let $\mathbf{k} !=k_{1} ! \cdots k_{n} !$ and $a_{\lambda}=a_{\lambda_{1}} \alpha_{\lambda_{2}} \cdots$ for any sequence $\left\{a_{i}: i \geq\right.$ $0\}$. The block matrix obtained from $\mathbf{A}$ by replacing each element $a_{i, j}$ with the $k_{i} \times k_{j}$ matrix consisting entirely of $a_{i, j}$ 's is denoted by $\mathbf{A}^{(\mathbf{k})}$ and is called the $\mathbf{k}$ - replication of $\mathbf{A}$. Let $\mathbf{Z}^{(\mathbf{k})}=\operatorname{diag}\left(z_{1,1}, \ldots, z_{1, k_{1}}, \ldots, z_{n, 1}, \ldots, z_{n, k_{n}}\right)$ and $\mathbf{Z}=\operatorname{diag}(\mathbf{z})=\mathbf{Z}^{(\mathbf{1})}$. For $\beta \subseteq \mathscr{N}_{n}=\{1, \ldots, n\}$, let $\mathbf{A}[\beta]$ be the submatrix of $\mathbf{A}$ with row and column labels in $\beta$. If the elements $m_{i, j} \in \mathrm{R}[[\mathbf{z}]]$ of an $n \times n$ matrix $\mathbf{M}$ have no constant terms, then a result of Jacobi, adapted to this ring, states that

$$
\text { trace } \log (\mathbf{I}-\mathbf{M})^{-1}=\log \operatorname{det}(\mathbf{I}-\mathbf{M})^{-1} .
$$

We review some results from the theory of symmetric functions. Let $\Lambda_{R}(\mathbf{y})=$ $\mathrm{R}\left[\left[y_{1}, y_{2}, \ldots\right]\right]^{\mathfrak{S}}$ denote the ring of symmetric functions in $y_{1}, y_{2}, \ldots$, with coefficient ring $\mathrm{R}$ where $\mathbf{y}=\left(y_{1}, y_{2}, \ldots\right)$. Where convenient, the name of this ring is abbreviated to $\Lambda$. It is graded by degree, so $\Lambda=\bigoplus_{i \geq 0} \Lambda^{(i)}$, where $\Lambda^{(i)}$ is the set of all symmetric functions of degree $i$ in $\mathbf{y}$. The elementary, complete, monomial, power sum and Schur symmetric functions are denoted by $e_{\lambda}(\mathbf{y}), h_{\lambda}(\mathbf{y}), m_{\lambda}(\mathbf{y}), p_{\lambda}(\mathbf{y})$, and $s_{\lambda}(\mathbf{y})$, respectively. Note that $\sum_{i \geq 0} h_{i} t^{i}=$ $\Pi_{j \geq 1}\left(1-t y_{j}\right)^{-1}=\left\{\sum_{i \geq 0} e_{i}(-t)^{i}\right\}^{-1}$. The Schur functions are given in terms of the complete symmetric functions by the Jacobi-Trudi identity

$$
s_{\lambda}(\mathbf{y})=\operatorname{det}\left[h_{\lambda_{i}-i+j}(\mathbf{y})\right]_{m \times m},
$$

where $m=l(\lambda)$. Cauchy's theorem states that

$$
\sum_{\nu} s_{\nu}\left(u_{1}, \ldots\right) s_{\nu}\left(v_{1}, \ldots\right)=\prod_{i, j \geq 1}\left(1-u_{i} v_{j}\right)^{-1},
$$

where the sum is over all partitions.

Let $\langle\cdot, \cdot\rangle$ be an inner product defined on $\Lambda_{R}$ by

$$
\left\langle h_{\lambda}, m_{\mu}\right\rangle=\delta_{\lambda, \mu},
$$

where $\lambda, \mu \vdash n$, and it follows that

$$
\left\langle s_{\lambda}, p_{\mu}\right\rangle=\chi_{\mu}^{\lambda} \quad \text { and } \quad\left\langle h_{\lambda}, s_{\mu}\right\rangle=K_{\lambda, \mu},
$$

where $K_{\lambda, \mu}$ are the Kostka numbers. The Schur functions are orthonormal with respect to this inner product. Let $\omega$ be the ring homomorphism defined by $\omega: \Lambda \rightarrow \Lambda: e_{k} \mapsto h_{k}$ extended linearly to $\Lambda$. Then $\omega\left(s_{\lambda}\right)=s_{\tilde{\lambda}}$ so $\omega$ is 
an isometry, and, from (2), $s_{\lambda}(\mathbf{y})=\operatorname{det}\left[e_{\tilde{\lambda}_{i}-i+j}(\mathbf{y})\right]_{\lambda_{1} \times \lambda_{1}}$. More generally, if $a_{0}, a_{1}, \ldots$ and $b_{0}, b_{1}, \ldots$ are sequences related by

$$
\sum_{k \geq 0} a_{k} t^{k}=\left\{\sum_{k \geq 0} b_{k}(-t)^{k}\right\}^{-1}
$$

then $\operatorname{det}\left[a_{\lambda_{i}-i+j}\right]_{m \times m}=\operatorname{det}\left[b_{\tilde{\lambda}_{i}-i+j}\right]_{\lambda_{1} \times \lambda_{1}}$. When $\lambda=\left[p^{q}\right] \vdash n$, this is a result due to Hadamard [4] concerning Hankel determinants.

\section{THE IMMANANT AS A DETERMINANT}

We begin by exploiting (5) in the enumeration of permutations to obtain the following result, which gives expressions for an arbitrary immanant.

Theorem 2.1. Let $\sum_{k} \Delta_{k} t^{k}=\operatorname{det}(\mathbf{I}-t \mathbf{Z A})^{-1}=\left\{\sum_{k} D_{k}(-t)^{k}\right\}^{-1}$. Then

(1) $\operatorname{Imm}_{\lambda} \mathbf{A}=\left[\mathbf{z}^{1}\right] \operatorname{det}\left[\Delta_{\lambda_{i}-i+j}\right]_{m \times m}$,

(2) $\operatorname{Imm}_{\lambda} \mathbf{A}=\left[\mathbf{z}^{1}\right] \operatorname{det}\left[D_{\tilde{\lambda}_{i}-i+j}\right]_{\lambda_{1} \times \lambda_{1}}$.

Proof. (1) We work in $\Lambda_{R}(y)$, where $R$ is an appropriately chosen coefficient ring whose choice will be clear from the context, so no further comment will be made about its selection. From (5),

$$
\operatorname{Imm}_{\lambda} \mathbf{A}=\left\langle s_{\lambda}(\mathbf{y}), \sum_{\sigma \in \mathfrak{S}_{n}} p_{\tau(\sigma)}(\mathbf{y}) \prod_{i=1}^{n} a_{i, \sigma(i)}\right\rangle .
$$

Now $\sum_{\sigma \in \mathfrak{S}_{n}} p_{\tau(\sigma)}(\mathbf{y}) \prod_{i=1}^{n} a_{i, \sigma(i)}$ is the (ordinary) generating function for permutations in $\mathfrak{S}_{n}$ with respect to cycle-type in which $a_{i, j}$ marks the occurrence of $i \mapsto j$ and $p_{k}(\mathbf{y})$ marks the occurrence of a cycle of length $k$. This is possible since the $p_{k}(\mathbf{y})$ are algebraically independent. Then the generating function for all cycles containing $\left\{\alpha_{1}, \ldots, \alpha_{i}\right\} \subseteq \mathscr{N}_{n}$ is

$$
\left[z_{\alpha_{1}} \cdots z_{\alpha_{i}}\right] \sum_{k \geq 1} \frac{1}{k} p_{k}(\mathbf{y}) \operatorname{trace}(\mathbf{Z A})^{k}
$$

Now a permutation is uniquely expressible as a product of disjoint (and therefore commuting) cycles, so it can be viewed as an unordered collection of cycles, the disjoint union of whose elements is $\mathscr{N}_{n}$. Thus

$$
\sum_{\sigma \in \mathfrak{S}_{n}} p_{\tau(\sigma)}(\mathbf{y}) \prod_{i=1}^{n} a_{i, \sigma(i)}=\left[\mathbf{z}^{\mathbf{1}}\right] \exp \sum_{k \geq 1} \frac{1}{k} p_{k}(\mathbf{y}) \operatorname{trace}\left((\mathbf{Z A})^{k},\right.
$$

so

$$
\begin{aligned}
\operatorname{Imm}_{\lambda} \mathbf{A} & =\left[\mathbf{z}^{\mathbf{1}}\right]\left\langle s_{\lambda}(\mathbf{y}), \exp \sum_{i \geq 1} \operatorname{trace} \log \left(\mathbf{I}-y_{i} \mathbf{Z A}\right)^{-1}\right\rangle \\
& =\left[\mathbf{z}^{\mathbf{1}}\right]\left\langle s_{\lambda}(\mathbf{y}), \prod_{i \geq 1} \operatorname{det}\left(\mathbf{I}-y_{i} \mathbf{Z A}\right)^{-1}\right\rangle,
\end{aligned}
$$

by (1); but $\prod_{i \geq 1} \operatorname{det}\left(\mathbf{I}-y_{i} \mathbf{Z A}\right)^{-1}=\sum_{\rho} \Delta_{\rho} m_{\rho}(\mathbf{y})$, where the sum is over all partitions. Substituting this and (2) into the expression for the immanant and 
then applying (4) and the bilinearity of the inner product gives

$$
\begin{aligned}
\operatorname{Imm}_{\lambda} \mathbf{A} & =\left[\mathbf{z}^{1}\right] \sum_{\sigma \in \mathfrak{S}_{m}} \operatorname{sgn}(\sigma) \sum_{\rho} \Delta_{\rho}\left\langle h_{\lambda-\delta_{m}+\sigma\left(\delta_{m}\right)}(\mathbf{y}), m_{\rho}(\mathbf{y})\right\rangle \\
& =\left[\mathbf{z}^{\mathbf{1}}\right] \sum_{\sigma \in \mathfrak{S}_{m}} \operatorname{sgn}(\sigma) \Delta_{\lambda-\delta_{m}+\sigma\left(\delta_{m}\right)}=\left[\mathbf{z}^{\mathbf{1}}\right] \operatorname{det}\left[\Delta_{\lambda_{i}-i+j}\right]_{m \times m} .
\end{aligned}
$$

(2) Direct from (1) and (6).

A special case of the MacMahon Master theorem now follows.

Corollary 2.2. $\operatorname{per} \mathbf{A}=\left[\mathbf{z}^{\mathbf{1}}\right] \operatorname{det}(\mathbf{I}-\mathbf{Z A})^{-1}$.

Proof. From Theorem 2.1(1), per $\mathbf{A}=\operatorname{Imm}_{[n]} \mathbf{A}=\left[\mathbf{z}^{\mathbf{1}} t^{n}\right] \operatorname{det}(\mathbf{I}-t \mathbf{Z A})^{-1}=$ $\left[\mathbf{z}^{1}\right] \operatorname{det}(\mathbf{I}-\mathbf{Z A})^{-1}$.

Corollary 2.2 enables us to reexpress Theorem 2.1(1) in terms of permanents.

Corollary 2.3. Let $\sum_{k} P_{k} t^{k}=\operatorname{per}(\mathbf{I}+t \mathbf{Z A})$. Then

$$
\operatorname{Imm}_{\lambda} \mathbf{A}=\left[\mathbf{z}^{1}\right] \operatorname{det}\left[P_{\lambda_{i}-i+j}\right]_{m \times m} .
$$

Proof. By Corollary 2.2, the squarefree terms in $\operatorname{det}(\mathbf{I}-t \mathbf{Z A})^{-1}$ agree with the squarefree terms in $\operatorname{per}(\mathbf{I}-t Z \mathbf{A})$, and the result follows from the Theorem 2.1 (1).

By specialising $\mathbf{A}$ it is possible to use these results directly to derive the familiar expressions for particular character evaluations. For example, let $\alpha=$ $\left[1^{a_{1}} 2^{a_{2}} \ldots\right], \tau(\sigma)=\alpha$, and $\omega_{n}$ be an $n$-cycle. Let $\mathbf{C}_{i}$ be the $i \times i\{0,1\}-$ matrix corresponding to an $i$-cycle, and let

$$
\mathbf{B}=\underbrace{\left(\mathbf{C}_{1} \oplus \cdots \oplus \mathbf{C}_{1}\right)}_{a_{1}} \oplus \cdots \oplus \underbrace{\left(\mathbf{C}_{n} \oplus \cdots \oplus \mathbf{C}_{n}\right)}_{a_{n}} .
$$

Then $\chi^{\left[1^{n}\right]}(\sigma)=\operatorname{Imm}_{\left[1^{n}\right]} \mathbf{B}, \chi^{[n]}(\sigma)=\operatorname{Imm}_{[n]} \mathbf{B}, \chi^{\alpha}\left(\omega_{n}\right)=\operatorname{Imm}_{\alpha} \mathbf{C}_{n}$. The details of evaluating these immanants are left to the reader.

By equating coefficients of $\prod_{i=1}^{n} a_{i, \sigma(i)}$ in Corollary 2.3, we obtain the expression $\sum_{\mu}\left\langle s_{\lambda}, m_{\mu}\right\rangle \cdot\left\langle h_{\mu}, p_{\tau(\sigma)}\right\rangle$ for $\chi^{\lambda}(\sigma)$; however, this expression is also an immediate consequence of (4) and (5).

The immanant can be also expressed as a Schur function at particular arguments. To see this, let $w_{1}, \ldots, w_{n}$ be the eigenvalues of the $n \times n$ matrix ZA. Then $1-y_{i} w_{j}$ are eigenvalues of $\mathbf{I}-y_{i} \mathbf{Z A}$ for $j=1, \ldots, n$, so from (3), $\prod_{i \geq 1} \operatorname{det}\left(\mathbf{I}-y_{i} \mathbf{Z A}\right)^{-1}=\prod_{i \geq 1} \prod_{j=1}^{n}\left(1-y_{i} w_{j}\right)^{-1}=\sum_{\mu} s_{\mu}(\mathbf{y}) s_{\mu}\left(w_{1}, \ldots, w_{n}\right)$. Since $\left\langle s_{\lambda}(\mathbf{y}), s_{\mu}(\mathbf{y})\right\rangle=\delta_{\lambda, \mu}$, the desired expression is, from (8),

$$
\operatorname{Imm}_{\lambda} \mathbf{A}=\left[\mathbf{z}^{1}\right] s_{\lambda}\left(w_{1}, \ldots, w_{n}\right) .
$$

\section{IMMANANTS OF $k$-REPLICATIONS}

In $\S 2$, expressions for $\operatorname{Imm}_{\lambda} \mathbf{A}$ were given as the coefficient of $\mathbf{z}^{\mathbf{1}}$ in various power series. The general coefficient is given by means of the following lemma for the k-replication of $\mathbf{A}$.

Lemma 3.1. If $\sum_{i \geq 0} D_{i}^{(\mathbf{k})} t^{i}=\operatorname{det}\left(\mathbf{I}+t \mathbf{Z}^{(\mathbf{k})} \mathbf{A}^{(\mathbf{k})}\right)$ and $f$ is a power series, then

$$
\left[z_{1,1} \cdots z_{1, k_{1}} \cdots z_{n, 1} \cdots z_{n, k_{n}}\right] f\left(D_{1}^{(\mathbf{k})}, D_{2}^{(\mathbf{k})}, \ldots\right)=\left[\mathbf{z}^{\mathbf{k}} / \mathbf{k} !\right] f\left(D_{1}, D_{2}, \ldots\right) .
$$


Proof. Let $\mathbf{U}=\operatorname{diag}\left(u_{1}, \ldots, u_{n}\right)$ and $u_{i}=z_{i, 1}+\cdots+z_{i, k_{i}}$. By expanding the determinant of the sum, we have $\operatorname{det}\left(\mathbf{I}+t \mathbf{Z}^{(\mathbf{k})} \mathbf{A}^{(\mathbf{k})}\right)=\operatorname{det}(\mathbf{I}+t \mathbf{U A})$. Then $\left[z_{1,1} \cdots z_{1, k_{1}} \cdots z_{n, 1} \cdots z_{n, k_{n}}\right] u_{1}^{k_{1}} \cdots u_{n}^{k_{n}}=\mathbf{k}$ ! , giving the result.

Comparing this with the results in $\S 2$ gives the following corollaries.

Corollary 3.2. Let $\lambda=\left(\lambda_{1}, \ldots, \lambda_{m}\right) \vdash N$ and $k_{1}+\cdots+k_{n}=N$. Then

(1) $\operatorname{Imm}_{\lambda} \mathbf{A}^{(\mathbf{k})}=\left[\mathbf{z}^{\mathbf{k}} / \mathbf{k} !\right] \operatorname{det}\left[\Delta_{\lambda_{i}-i+j}\right]_{m \times m}$,

(2) $\operatorname{Imm}_{\lambda} \mathbf{A}^{(\mathbf{k})}=\left[\mathbf{z}^{\mathbf{k}} / \mathbf{k} !\right] \operatorname{det}\left[D_{\tilde{\lambda}_{i}-i+j}\right]_{\lambda_{1} \times \lambda_{1}}$.

Proof. (2) From Theorem 2.1(2),

$$
\operatorname{Imm}_{\lambda} \mathbf{A}^{(\mathbf{k})}=\left[z_{1,1} \cdots z_{1, k_{1}} \cdots z_{n, 1} \cdots z_{n, k_{n}}\right] \operatorname{det}\left[D_{\tilde{\lambda}_{i}-i+j}^{(\mathbf{k})}\right] \lambda_{\lambda_{1} \times \lambda_{1}} .
$$

The result follows from Lemma 3.1.

(1) This follows from Theorem 2.1(1) and Lemma 3.1, since a power series in the $\Delta_{i}$ is a power series in the $D_{i}$, from (6).

An immediate consequence is the following.

Theorem 3.3 (MacMahon Master theorem).

$$
\left[\mathbf{z}^{\mathbf{k}}\right] \prod_{i=1}^{n}\left(\sum_{j=1}^{n} a_{i, j} z_{j}\right)^{k_{i}}=\left[\mathbf{z}^{\mathbf{k}}\right] \operatorname{det}(\mathbf{I}-\mathbf{Z A})^{-1} .
$$

Proof. From Corollary 3.2(1), with $\lambda=[N]$, we have

$$
\mathbf{k} !\left[\mathbf{z}^{\mathbf{k}}\right] \prod_{i=1}^{n}\left(\sum_{j=1}^{n} a_{i, j} z_{j}\right)^{k_{i}}=\left[\mathbf{z}^{\mathbf{k}} / \mathbf{k} !\right] \operatorname{det}(\mathbf{I}-\mathbf{Z A})^{-1},
$$

since both are equal to per $\mathbf{A}^{(\mathbf{k})}$. The factor $\mathbf{k}$ ! arises since, in the permanent, the columns are distinguishable.

Let $\mathbf{l}=\left(l_{1}, \ldots, l_{n}\right)$ and $l_{1}+\cdots+l_{n}=k_{1}+\cdots+k_{n}$. The $(\mathbf{k}, \mathbf{l})$-replication of $\mathbf{A}$ is the matrix obtained from $\mathbf{A}$ by replacing each $a_{i, j}$ with a $k_{i} \times l_{j}$ block of $a_{i, j}$ 's and is denoted by $\mathbf{A}^{(\mathbf{k}, \mathbf{l})}$. From [5], the exponential generating function for $\mathbf{k}$, l-replications of the permanent of $\mathbf{A}$ is

$$
\sum_{\mathbf{k}, 1 \geq \mathbf{0}} \operatorname{per} \mathbf{A}^{(\mathbf{k}, 1)} \frac{\mathbf{x}^{\mathbf{k}} \mathbf{y}^{\mathbf{I}}}{\mathbf{k} ! ! !}=\exp \mathbf{x A y} \mathbf{y}^{t}
$$

where $\mathbf{x}=\left(x_{1}, \ldots, x_{n}\right)$. We have been unable to find an extension of this result to the case of an arbitrary immanant, although Corollary 3.2 serves this purpose for the "diagonal" of this series.

Finally, we consider

$$
\operatorname{Imm}_{\lambda}^{(u)} \mathbf{A}=\sum_{\sigma \in \mathfrak{S}_{n}} \chi^{\lambda}(\sigma) u^{l(\tau(\sigma))} \prod_{i=1}^{n} a_{i, \sigma(i)},
$$

a generalisation of the immanant in which an indeterminate $u$ marks the number of (disjoint) cycles. 
Corollary 3.4. Let $\sum_{k} \Delta_{k}^{(u)} t^{k}=\operatorname{det}(\mathbf{I}-t \mathbf{Z A})^{-u}=\left\{\sum_{k} D_{k}^{(u)}(-t)^{k}\right\}^{-1}$. Then

(1) $\operatorname{Imm}_{\lambda}^{(u)} \mathbf{A}^{(\mathbf{k})}=\left[\mathbf{z}^{\mathbf{k}} / \mathbf{k}\right.$ ! $] \operatorname{det}\left[\Delta_{\lambda_{i}-i+j}^{(u)}\right]_{m \times m}$,

(2) $\operatorname{Imm}_{\lambda}^{(u)} \mathbf{A}^{(\mathbf{k})}=\left[\mathbf{z}^{\mathbf{k}} / \mathbf{k} !\right] \operatorname{det}\left[D_{\tilde{\lambda}_{i}-i+j}^{(u)}\right]_{\lambda_{1} \times \lambda_{1}}$.

Proof. Follows from the methods of proof of Theorem 2.1 and Corollary 3.2, having replaced (7) by

$$
\operatorname{Imm}_{\lambda}^{(u)} \mathbf{A}=\left[\mathbf{z}^{\mathbf{1}}\right]\left\langle s_{\lambda}(\mathbf{y}), \exp \left\{u \sum_{i \geq 1} \operatorname{trace} \log \left(\mathbf{I}-y_{i} \mathbf{Z A}\right)^{-1}\right\}\right\rangle .
$$

The special case $\lambda=[n]$ of Corollary 3.4 has been previously obtained combinatorially by Foata and Zeilberger [1].

\section{LitTLEWOOdS's RESUlts}

The results that we have given are related to the following little known theorems of Littlewood [7, §6.5].

Theorem I [7, p. 118]. Corresponding to any relation between S-functions of total weight $n$, we may replace each $S$-function by the corresponding immanant of complementary coaxial minors of $\left[a_{s, t}\right]$, provided that every product is summed for all sets of complementary coaxial minors.

In the following two theorems, Littlewood generalised the concept of a minor to permit arbitrary repetition of rows and columns. Thus A has $\left(\begin{array}{c}n-r+1 \\ r\end{array}\right)^{2} r$ rowed minors. He also attached a factor of $1 / r$ ! to every immanant of a minor for each row that is repeated $r$ times in the minor.

Theorem II [7, p. 120]. Corresponding to any relation between $S$-functions we may replace each $S$-function by the corresponding immanant of a coaxial minor of $\left[a_{s t}\right]$, provided that we sum with respect to all coaxial minors of the appropriate order.

Theorem III [7, p. 121]. The S-function $\{\lambda\}$ of weight $p$ of the characteristic roots of a matrix $\left[a_{s t}\right]$ is equal to the sum of immanants corresponding to the partition $\{\lambda\}$ of all p-rowed coaxial minors of $\left[a_{s t}\right]$.

The next proposition is an alternative presentation of Theorem 2.1 adapted to the action of $\phi_{\mathbf{A}}$, the linear mappings defined on $\Lambda^{(n)}$ by $\phi_{\mathbf{A}} g(\mu) p_{\mu}=$ $\sum_{\sigma \in \mathscr{C}_{\mu}} \prod_{i=1}^{n} a_{i, \sigma(i)}$, where $\mathscr{C}_{\mu}$ is the conjugacy class indexed by $\mu \vdash n$ and $g(\mu)=\left|\mathscr{C}_{\mu}\right| / n !$.

Proposition 4.1. Let $f \in \mathrm{R}\left[\left[h_{1}, h_{2}, \ldots\right]\right]$ and $g \in \mathrm{R}\left[\left[e_{1}, e_{2}, \ldots\right]\right]$. Then

(1) $\phi_{\mathbf{A}} s_{\lambda}=\operatorname{Imm}_{\lambda} \mathbf{A}$,

(2) $\phi_{\mathbf{A}} f\left(h_{1}, h_{2}, \ldots\right)=\left[\mathbf{z}^{\mathbf{1}}\right] f\left(P_{1}, \ldots\right)=\left[\mathbf{z}^{\mathbf{1}}\right] f\left(\Delta_{1}, \ldots\right)$,

(3) $\phi_{\mathbf{A}} g\left(e_{1}, e_{2}, \ldots\right)=\left[\mathbf{z}^{\mathbf{1}}\right] g\left(D_{1}, \ldots\right)$.

Proof. (1) Follows from (5).

(2) and (3) These follow by adapting the proof of Theorem 2.1 to show that $\phi_{\mathbf{A}} h_{\lambda}=\left[\mathbf{z}^{1}\right] \Delta_{\lambda}=\left[\mathbf{z}^{1}\right] P_{\lambda}$ and that $\phi_{\mathbf{A}} e_{\lambda}=\left[\mathbf{z}^{1}\right] D_{\lambda}$, and by then using the linearity of $\phi_{\mathbf{A}}$. 
Lemma 4.2. Let $\alpha \vdash l$ and $\beta \vdash m$. Then, with the convention that the unions that appear below are to be disjoint,

$$
\phi_{\mathbf{A}} s_{\lambda} s_{\mu}=\sum_{\substack{\alpha \cup \beta=\mathscr{N}_{n} \\|\alpha|=l,|\beta|=m}} \phi_{\mathbf{A}[\alpha]} s_{\lambda} \phi_{\mathbf{A}[\beta]} s_{\mu} .
$$

Proof. From Proposition 4.1(2) and the Jacobi-Trudi identity,

$$
\begin{aligned}
\phi_{\mathbf{A}} s_{\lambda} s_{\mu} & =\left[\mathbf{z}^{\mathbf{1}}\right] \operatorname{det}\left[P_{\lambda_{i}-i+j}\right]_{l \times l} \operatorname{det}\left[P_{\mu_{i}-i+j}\right]_{m \times m} \\
& =\sum_{\alpha \cup \beta=\mathscr{N}_{n}}\left[z_{\alpha}\right] \operatorname{det}\left[P_{\lambda_{i}-i+j}\right]_{l \times l}\left[z_{\beta}\right] \operatorname{det}\left[P_{\mu_{i}-i+j}\right]_{m \times m} \\
& =\sum_{\alpha \cup \beta=\mathscr{N}_{n}} \operatorname{Imm}_{\lambda} \mathbf{A}[\alpha] \operatorname{Imm}_{\mu} \mathbf{A}[\beta] \quad \text { (from Corollary 2.3), }
\end{aligned}
$$

and the result follows from Proposition 4.2(1).

More generally, if $f \in \Lambda^{(i)}, g \in \Lambda^{(n-i)}$, then it follows immediately that

$$
\phi_{\mathbf{A}} f g=\sum_{\substack{|\alpha| \subseteq \mathscr{N}_{m} \\|\alpha|=i}} \phi_{\mathbf{A}[\alpha]} f \phi_{\mathbf{A}\left[\mathscr{N}_{n}-\alpha\right]} g .
$$

Theorem I, in which Littlewood used the terms " $S$-function" for Schur function and "coaxial minor" for principal minor, is obtained by applying Lemma 4.2 to an arbitrary relation between Schur functions of total weight $n$. In particular, Lemma 4.2 contains Theorem 2.1 as a special case. The appearance of principal minors and the role of complementarity is clear.

Littlewood's conventions for Theorems II and III entail k-replications. Although Corollary 3.2 follows by this means from Theorem II, it is not clear how Littlewood would have handled Corollary 3.4.

Remark (9) follows from Theorem III by replacing each $a_{i, j}$ with $z_{i} a_{i, j}$ and by applying $\left[\mathbf{z}^{1}\right]$. Littlewood's convention allows repeated rows, so the extraction of the linear term ensures that each row appears exactly once.

Note that Corollary 2.2 is recovered by applying Theorem I to the relation $\sum_{i=0}^{n} e_{i} h_{n-i}(-1)^{i}=0, \quad n>0$ with the identification of $e_{k}=s_{\left[1^{k}\right]}$ and $h_{k}=$ $s_{[k]}$. Littlewood [7, p. 119] did this for $n=4$. Had Littlewood given an example of the use of Theorem II of the type he gave for Theorem I with $n=4$, he would have obtained an instance of the MacMahon Master theorem involving the coefficient of the general term $x_{1}^{k_{1}} \cdots x_{4}^{k_{4}}$ rather than the coefficient of $x_{1} \cdots x_{4}$. It therefore appears that Littlewood [6] had, implicitly, a symmetric function proof of the MacMahon Master theorem. Merris and Watkins [10] have used Theorem I to obtain bounds for generalised matrix functions.

\section{The Young IDEMPotents}

The action of the mapping $\phi_{\mathbf{A}}$, defined in $\S 4$, is related to certain idempotents in $\mathbb{C S}_{n}$, for which Young [11] gave an explicit construction. Any $f \in \mathbb{C S}_{n}$ may be regarded as a function $f: \mathfrak{S}_{n} \rightarrow \mathbb{C}$ or as a formal sum $\sum_{\sigma \in \mathfrak{S}_{n}} f(\sigma) \sigma$. The centre $\mathscr{Z}_{n}$ is the set of all class functions so $\left\{\mathrm{K}_{\lambda}: \lambda \vdash n\right\}$ is a basis of $\mathscr{Z}_{n}$, where $\mathrm{K}_{\lambda}=\sum_{\sigma \in \mathscr{C}_{\lambda}} \sigma$. Also, $\chi^{\lambda} \in \mathscr{Z}_{n}$. Let $\psi_{\mathbf{A}} \sigma=\prod_{i=1}^{n} a_{i, \sigma(i)}$, extended linearly to $\mathbb{C S}_{n}$. Then $\psi_{\mathrm{A}} \chi^{\lambda}=\operatorname{Imm}_{\lambda} \mathbf{A}$. But the Frobenius map is $F: \mathscr{Z}_{n} \rightarrow \Lambda^{(n)}: \mathrm{K}_{\mu} \mapsto g(\mu) p_{\mu}$, so $\phi_{\mathbf{A}}=F^{-1} \psi_{\mathbf{A}}$ and, from the above, 
$\chi^{\lambda}=F^{-1} s_{\lambda}$. Let $\mathrm{p}^{\lambda}=F^{-1} h_{\lambda}$ and $\mathrm{n}^{\lambda}=F^{-1} e_{\lambda}$. Then $\mathrm{p}^{\lambda}, \mathrm{n}^{\lambda}, \chi^{\lambda}$ are idempotents. From $\S 2$, these have power series representations

$$
\psi_{\mathbf{A}} \mathrm{p}^{\lambda}=\left[\mathbf{z}^{1}\right] \Delta_{\lambda}, \quad \psi_{\mathbf{A}} \mathrm{n}^{\lambda}=\left[\mathbf{z}^{1}\right] D_{\lambda}, \quad \psi_{\mathbf{A}} \chi^{\lambda}=\left[\mathbf{z}^{1}\right] \operatorname{det}\left[\Delta_{\lambda_{i}-i+j}\right]=\left[\mathbf{z}^{1}\right] \operatorname{det}\left[D_{\tilde{\lambda}_{i}-i+j}\right]
$$

and are constructed as follows [11]. Let $\lambda \vdash n$, be an arbitrary (ordered) partition of $\mathscr{N}_{n}$ whose $i$ th block has size $\lambda_{i}$, for $i=1, \ldots, m$, and Fix $\pi$ be the set of all permutations in $\mathfrak{S}_{n}$ that fix every block of $\pi$. Thus Fix $\pi \cong \mathfrak{S}_{\lambda_{1}} \times \cdots \times$ $\mathfrak{S}_{\lambda_{m}}$. Let $\lambda !=\lambda_{1} ! \ldots \lambda_{m} !, \mathrm{P}_{\pi}=\sum_{\sigma \in \mathrm{Fix} \pi} \sigma / \lambda !$, and $\mathrm{N}_{\pi}=\sum_{\sigma \in \mathrm{Fix} \pi} \operatorname{sgn}(\sigma) w \sigma / \lambda !$, and $\mathrm{N}_{\pi}=\sum_{\sigma \in \mathrm{Fix} \pi \operatorname{sgn}(\sigma)} \operatorname{sgn}(\sigma) \sigma / \lambda !$. If $\mathscr{T}$ is any Young tableau of shape $\lambda$, its rows and columns, respectively, induce (set) partitions $\rho, \kappa$ of $\mathscr{N}_{n}$ whose block sizes are listed by $\lambda, \tilde{\lambda}$. Then

$$
\begin{aligned}
& \mathrm{p}^{\lambda}=\sum_{\sigma \in \mathfrak{S}_{n}} \sigma \mathrm{P}_{\pi} \sigma^{-1}, \quad \mathrm{n}^{\lambda}=\sum_{\sigma \in \mathfrak{S}_{n}} \sigma \mathrm{N}_{\pi} \sigma^{-1}, \\
& \chi^{\lambda}=\frac{1}{n !} \lambda ! \tilde{\lambda} ! \chi^{\lambda}(e) \sum_{\sigma \in \mathfrak{S}_{n}} \sigma \mathrm{P}_{\rho} \mathrm{N}_{\kappa} \sigma^{-1},
\end{aligned}
$$

independent of the choices of $\pi$ and $\mathscr{T}$.

It would be of interest to derive the power series representations (10) for $\psi_{\mathrm{A}} \chi^{\lambda}$ from Young's constructions, but we have been unable to do so.

\section{ACKNOWLEDGMENTS}

We thank Ian Macdonald for pointing out that (9) follows from (8).

\section{REFERENCES}

1. D. Foata and D. Zeilberger, Laguerre polynomials, weighted derangements, and positivity, SIAM. J. Discrete Math. 1 (1988), 425-433.

2. A. M. Garsia and J. Remmel, Symmetric functions and raising operators, Linear and Multilinear Algebra 10 (1981), 15-43.

3. I. P. Goulden and D. M. Jackson, Immanants of combinatorial matrices, J. Algebra (to appear).

4. J. S. Hadamard, Essai sur l'étude des fonctions données par leur développement de Taylor, Journal des Mathématiques (4), 8 (1892), 1-86.

5. D. M. Jackson, The unification of certain enumeration problems for sequences, J. Combin. Theory Ser. A 22 (1977), 92-96.

6. D. E. Littlewood, Invariant theory, tensors and group characters, Philos. Trans. Roy. Soc. London Ser. A 239 (1944), 305-365.

7. _ The theory of group characters, Clarendon Press, Oxford, 1950.

8. I. G. Macdonald, Symmetric functions and Hall polynomials, Clarendon Press, Oxford, 1979.

9. P. A. MacMahon, Combinatory analysis, Chelsea, New York, 1960.

10. R. Merris and W. Watkins, Inequalities and identities for generalized matrix functions, Linear Algebra Appl. 64 (1985), 223-242.

11. G. de B. Robinson The collected papers of Alfred Young: 1873-1940, Math. Exp. No. 21, University of Toronto, 1977.

Dept. of Combinatorics and Optimization, University of Waterloo, Waterloo OnTARIO, CANADA N2L 3G1

Dept. of Combinatorics and Optimization, University of Waterloo, Waterloo ONTARIO, CANADA N2L 3G1 